\title{
Synchronization Techniques Comparison for Sensorless Control applied to PMSG
}

\author{
L. G. González ${ }^{1}$, E. Figueres ${ }^{2}$, G. Garcerá2 ${ }^{2}$ O. Carranza ${ }^{3}$ \\ ${ }^{1}$ Departamento de Electrónica y Comunicaciones, Universidad de los Andes, Mérida, Venezuela. \\ Phone:+0058 02742402907, e-mail: lgerardo@ula.ve, \\ ${ }^{2}$ Departamento de Ingeniería Electrónica - UPV, Camino de Vera S/N, 7F, Valencia, 46022, España. \\ Phone:+0034963876001, e-mail: efiguere@eln.upv.es, ggarcera@eln.upv.es
}

\begin{abstract}
${ }^{3}$ Escuela Superior de Cómputo - Instituto Politécnico Nacional, Av. Juan de Dios Bátiz S/N, D. F., 07738, México. Phone:+005255576000, Ext: 52020 , e-mail: ocarranzac@ipn.mx
\end{abstract}

\begin{abstract}
This paper encompasses a comparative study of techniques for position and speed estimation applied to permanent magnet synchronous generators (PMSG), connected to the distribution grid by means of a back to back converter. In the context of low and medium power wind energy conversion systems, the robustness of the sensorless techniques are studied taking into account the tolerance of the PMSG parameters.
\end{abstract}

\section{Keywords}

Sensorless Control, Extended Kalman Filter, Sliding Mode Observer, PLL, Simplified Kalman Observer, Permanent Magnet Synchronous Generators.

\section{1.- Introduction}

The use of PMSGs has special interest in low power wind energy applications, due to their small size and high power density. PMSGs operate in a large speed range, so that it is mandatory to measure or estimate their speed and position. In small and medium power applications it is preferred the use of sensorless techniques in order to reduce the implementation cost, allowing vector control with a position and speed machine estimation. The general system performance strongly depends on the chosen estimation technique.

About the control strategies, it is necessary to emphasize that in systems with generator position and speed sensors the wind energy conversion efficiency ranges between $56 \%$ and $63 \%$, whereas with sensorless techniques that efficiency is usually between $55 \%$ and $61 \%$. In low power systems that conversion efficiency reduction is generally acceptable, taking into account the cost and complexity reduction achieved by sensorless control.
In this work the performance of several estimation techniques is studied, like the sliding mode observer (SMO), the synchronous reference frame three-phase phase locked loop (PLL), the linear observer with extended Kalman filter (EKF) and its simplified implementation, called the simplified Kalman observer (SKO). A comparative study has been carried out, allowing to select a technique which increases the overall system performance.

The system topology used in this article is based in a PMSG, controlled by a back to back converter, as shown in figure 1. This topology shows a better performance compared to the PMSG connected through a diode bridge rectifier and a boost converter to the DC-link for the small and medium power range [1]. The back to back converter transfers the energy generated by the PMSG to the grid. The chosen control strategy is the zero reactive current, which allows to achieve the maximum system performance; with this control technique the active and reactive currents are controlled by PI regulators. For the active current control loop, the reference will be obtained by the maximum power point tracker (MPPT) algorithm. It is worth to point out that the goal is getting more torque-current ratio, which depends on the estimated position signal.

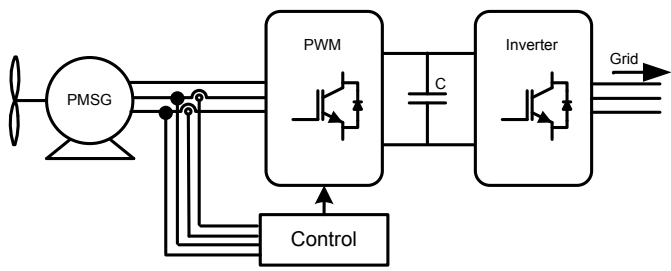

Fig. 1. Wind energy system under study. 


\section{2.- Permanent Magnet Synchronous Generators (PMSG) Model}

The one phase equivalent circuit of a PMSG with a sinusoidal flux distribution is modeled in [2] and shown in figure 2, following equation (1), where: $R_{s}$ and $L_{s}$ are the stator internal resistance and inductance, $u_{s}$ is the terminal voltage and $e_{a}$ is the electromotive force (EMF) generated by the permanent magnets. Equations (2) and (3) show the PMSG model in a synchronous reference frame (Park), $d q$, figure 3 .

$$
\begin{gathered}
\frac{d i_{a}}{d t}=-\frac{R_{s}}{L_{s}} i_{a}+\frac{e_{a}}{L_{s}}-\frac{u_{s}}{L_{s}} \\
u_{s d}=-R_{s} i_{s d}+\omega_{e} \psi_{s q}-\dot{\psi}_{s d} \\
u_{s q}=-R_{s} i_{s q}-\omega_{e} \psi_{s d}-\dot{\psi}_{s q}
\end{gathered}
$$

The induced flux linkages in the stator ( $\mathrm{dq}$ frame) are described by (4) and (5):

$$
\begin{aligned}
& \psi_{s d}=L_{d} i_{s d}-\Psi_{P M} \\
& \psi_{s q}=L_{q} i_{s q}
\end{aligned}
$$

In the former equations $\omega_{\mathrm{e}}$ is the electric angular frequency of the PMSG, whereas $\Psi_{\mathrm{PM}}$ is the flux linkage in the permanent magnets. $u_{s d}$ and $u_{s q}$ are the terminal

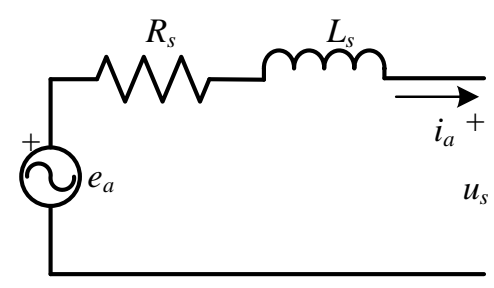

Fig.2 Equivalent circuit for phase (PMSG)

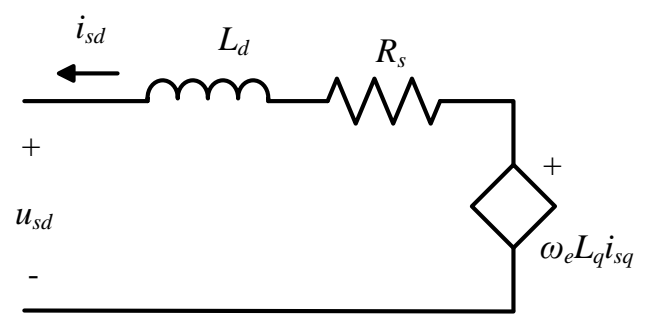

Fig. 3a

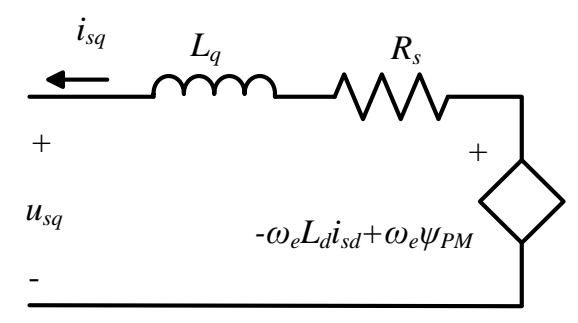

Fig. 3b

Fig. 3. Equivalent Circuit of a PMSG in the synchronous reference frame (a) d-axis, (b) q-axis. voltages (dq frame), $i_{s d}$ and $i_{s q}$ are the generator dq frame currents, and $L_{d}$ and $L_{q}$ are the stator inductances in the $\mathrm{dq}$ frame. The electrical torque applied to the PMSG rotor is shown in (6). If the PMSG is taken without rotor saliency (where $L_{d}=L_{q}=L$ ), the electrical torque is simplified following (7),

$$
\begin{gathered}
T_{e}=\frac{3}{2} \frac{P}{2}\left[\left(L_{d}-L_{q}\right) i_{s d} i_{s q}-\Psi_{P M} i_{s q}\right] \\
T_{e}=\frac{3}{2} \frac{P}{2}\left[\Psi_{P M} i_{s q}\right]
\end{gathered}
$$

where $P$ is the number of poles.

\section{3.- Position and Speed estimation using the Extended Kalman Filter (EKF).}

The rotor position and speed of a PMSG are related to the electromotive force (EMF). One of the most common techniques used for estimation of these variables is the extended Kalman filter observer, which can be applied using the framework represented in figure 4 . The lineal observer has been built up starting the machine model equations, taking into account the implicit tolerances of the model and estimation parameters of the PMSG. The discrete model and the Kalman filter structure adapted to a PMSG are shown in [3]. The Kalman filter matrices are shown in the following.

The discrete model of the estimator of the EMF is determined by (8),

$$
\left\{\begin{array}{c}
x(k+1)=A_{d} x(k)+B_{d} u(k) \\
y(k)=C_{d} x(k)
\end{array}\right\}
$$

where $x$ is the estimated signal vector (9), whereas $u$ is the synchronous machine phase voltage vector, and $y$ is the estimated line output currents, both in the fixed (Clark) reference frame $\alpha \beta$.

$$
x=\left[\begin{array}{c}
i_{s d} \\
i_{s q} \\
\omega_{e} \\
\theta
\end{array}\right], y=\left[\begin{array}{c}
i_{\alpha} \\
i_{\beta}
\end{array}\right], u=\left[\begin{array}{l}
u_{\alpha} \\
u_{\beta}
\end{array}\right]
$$

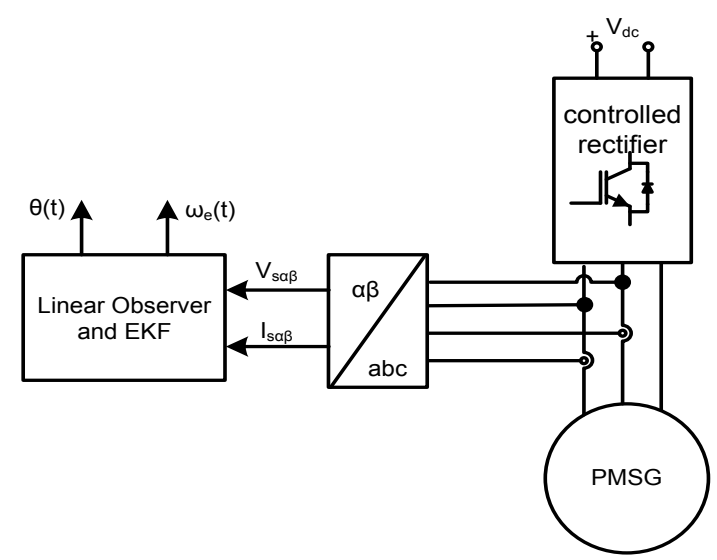

Fig. 4, Linear Observer and EKF 
The extended discrete model is shown in (10):

$$
\left\{\begin{array}{c}
x(k+1)=A_{d} x(k)+B_{d} u(k)+v(k) \\
y(k)=C_{d} x(k)+w(k)
\end{array}\right\}
$$

Where the matrixes of the discrete model depend on the sampling time $\left(T_{s}\right)$ and are expressed in $(11,12,13)$

$$
\begin{gathered}
A_{d}=\left[\begin{array}{cccc}
1-\frac{R}{L} \cdot T_{S} & \omega_{e} \cdot T_{s} & 0 & 0 \\
-\omega_{e} \cdot T_{s} & 1-\frac{R}{L} \cdot T_{S} & \frac{\Psi_{P M}}{L} \cdot T_{S} & 0 \\
0 & 0 & 1 & 0 \\
0 & 0 & T_{s} & 1
\end{array}\right] \\
B_{d}=\left[\begin{array}{cc}
\frac{T_{S}}{L} \cos (\theta) & -\frac{T_{S}}{L} \operatorname{sen}(\theta) \\
-\frac{T_{s}}{L} \operatorname{sen}(\theta) & \frac{T_{S}}{L} \cos (\theta) \\
0 & 0 \\
0 & 0
\end{array}\right] \\
C_{d}=\left[\begin{array}{cccc}
\cos (\theta) & -\operatorname{sen}(\theta) & 0 & 0 \\
\operatorname{sen}(\theta) & \cos (\theta) & 0 & 0
\end{array}\right]
\end{gathered}
$$

The terms $v(k)$ and $w(k)$ represent process and measurement noises, respectively. We can assume that these are independent white noises with a normal distribution performance and zero mean value. Their probability functions are expressed in the following, :

$$
p(v) \sim N(0, Q) \quad p(w) \sim N(0, R)
$$

where $Q$ and $R$ are the covariance matrixes of the $v(k)$ and $w(k)$ noises. There also exists a third matrix, $P$, which represents a covariance error of the state estimation.

The EKF algorithm shown in [3] has the following operation sequence:

\section{1.- Initialization}

2.- State vector prediction

$$
x(k+1 \mid k)=x(k+1)=A_{d} \hat{x}(k)+B_{d} u(k)
$$

3.- Estimation of the covariance matrix of the state prediction:

$$
P(k+1)=f(k+1) \cdot P(k) f^{T}(k+1)+Q
$$

Where $f(k+1)$ is:

$$
f(k+1)=\left.\frac{\partial\left[A_{d} x(k)+B_{d} u(k)\right]}{\partial x}\right|_{x=\hat{x}(k+1)}
$$

4.- Gain matrixes calculus:

$$
\begin{array}{r}
K(k+1)=P(k+1) \cdot h^{T}(k+1) \cdot[h(k+1) \cdot P(k+ \\
\left.1) \cdot h^{T}(k+1)+R\right]^{-1}
\end{array}
$$

Where $h(k+1)$ is:

$$
h(k+1)=\left.\frac{\partial\left[C_{d}(k) x\right]}{\partial x}\right|_{x=\hat{x}(k+1)}
$$

5.-State vector estimation:

$$
\begin{aligned}
\hat{x}(k+1)=x(k+1)+K(k+1) \\
\cdot\left[y(k+1)-C_{d}(k+1) \cdot x(k+1)\right]
\end{aligned}
$$

6.- Estimation correction of the covariance matrix:

$$
\hat{P}(k+1)=P(k+1)-K(k+1) \cdot h(k+1) \cdot P(k+1)
$$

7.- Data actualization:

$$
k=k+1, \quad x(k)=x(k-1), \quad P(k)=P(k-1)
$$

8.- Start a new iteration and go back to step 2. In this case study the following covariance matrixes $Q, R$ where used:

$$
\begin{gathered}
Q=\left[\begin{array}{cccc}
0.5 & 0 & 0 & 0 \\
0 & 0.5 & 0 & 0 \\
0 & 0 & 2 & 0 \\
0 & 0 & 0 & 0.05
\end{array}\right] \\
R=\left[\begin{array}{cc}
0.05 & 0 \\
0 & 0.05
\end{array}\right]
\end{gathered}
$$

\section{4.- Position and Speed estimation using the Simplified Kalman Observer (SKO).}

A simplified version of the extended Kalman filter, the Extended Kalman Observer (SKO) was proposed in [4]. The simplified Kalman filter has a variable reduced state vector variable and a constant gain matrix. Therefore, the real time programming of the simplified version is easier than the EKF version, which needs a high computational effort. In the particular case of the PMSG, the SKO has as input variables the estimation of the induced electromotive force (IEMF in the stationary $\alpha \beta$ reference frame $\left(e_{\alpha}(k), e_{\beta}(k)\right)$, defined by a linear estimator following equation (1). The filter is reduced to equations (14), where $\hat{\theta}(k), \widehat{\omega}_{e}(k)$, are the rotor position and EMF speed, whereas $w(k)$, is an auxiliary variable which represents the estimated error.

$$
\left\{\begin{array}{c}
\varepsilon(k)=e_{\alpha}(k) \cos \hat{\theta}(k)-e_{\beta}(k) \sin \hat{\theta}(k) \\
\hat{\theta}(k+1)=\left[\hat{\theta}(k)+T_{s} \widehat{\omega}_{e}(k)+k_{e 1} \varepsilon(k)\right] \\
\widehat{\omega}_{e}(k+1)=\widehat{\omega}_{e}(k)+\dot{w}(k)+k_{e 2} \varepsilon(k) \\
\dot{w}(k+1)=\dot{w}(k)+k_{e 3} \varepsilon(k)
\end{array}\right\}
$$

The filter gains $k_{e 1}=0.0038, k_{e 2}=0.7357$ and $k_{e 3}=0.0007$, are tuning parameters, which are calculated using the recursive algorithm used in the EKF. In this case the matrix gain is determined with the $d q l r \mathrm{MATLAB}^{\mathrm{TM}}$ function [10]. It is worth to point out that the estimator gain doesn't depend on the system parameters. 


\section{5.- The Three-Phase PLL (PLL).}

The three-phase pll is also called DQPLL or SRFPLL, because it operates on a $d q$ synchronous reference frame, using closed loop control to determine in a simple way if a signal is in phase with a reference signal [5]. In this technique the reference signals are the induced flux in the machine, determined by equation (15), which is the EMF integral in the three phase stationary frame reference system $a b c$. The response of the PLL to harmonics and high frequency switching noise at the PMSG terminals is remarkable. The PLL scheme is shown in figure 5.

$$
\Psi_{a}(t)=\int\left(e_{a}(t)\right) d t
$$

The tuning elements of the PLL observer are $K_{p}=210$, $K_{i}=5095$, in order to obtain crossover frequency of $30 \mathrm{~Hz}$.

\section{6.- Sliding Mode Observer (SMO)}

The Sliding Mode Observer technique estimates the EMF in the synchronous generator and allows to directly estimate the rotor position [6]. The main observer equation is shown by (16), where $\hat{i}_{\alpha}, \hat{i}_{\beta}$ are the estimated currents in the fixed $\alpha \beta$ reference frame (Clark), $v_{\alpha}, v_{\beta}$, are the machine phase to phase voltages, and $z_{\alpha}, z_{\beta}$ are the electromotive forces estimation in the generator. Parameters $z_{\alpha}, z_{\beta}$ are determined by equation (17), where: $i_{\alpha}, i_{\beta}$ are the generator currents, $e_{\alpha}, e_{\beta}$ are the low-frequency components of the electromotive force induced in the generator, $K$ is the observer gain, which is defined by a Lyapunov stability function. Some researches analyze the sub-oscillations reduction by replacing the sign function by others like the saturation or sigmoid functions [7][8][9]. Finally, with the lowfrequency component of the estimated EMF it is obtained the equation (17) and the estimated position (18).

$$
\begin{gathered}
\frac{d}{d t}\left[\begin{array}{l}
\hat{i}_{\alpha} \\
\hat{i}_{\beta}
\end{array}\right]=-\frac{R_{s}}{L_{s}}\left[\begin{array}{l}
\hat{i}_{\alpha} \\
\hat{i}_{\beta}
\end{array}\right]+\frac{1}{L_{s}}\left[\begin{array}{l}
Z_{\alpha} \\
z_{\beta}
\end{array}\right]-\frac{1}{L_{s}}\left[\begin{array}{l}
u_{\alpha} \\
u_{\beta}
\end{array}\right] \\
{\left[\begin{array}{l}
Z_{\alpha} \\
z_{\beta}
\end{array}\right]=K \cdot \operatorname{sgn}\left(\left[\begin{array}{l}
i_{\alpha} \\
i_{\beta}
\end{array}\right]-\left[\begin{array}{l}
\hat{i}_{\alpha} \\
\hat{i}_{\beta}
\end{array}\right]\right) \approx\left[\begin{array}{l}
e_{\alpha} \\
e_{\beta}
\end{array}\right]} \\
\hat{\theta}(t)=\operatorname{Arctan}\left(\frac{e_{\alpha}(t)}{e_{\beta}(t)}\right)
\end{gathered}
$$

In this application $K=500$, and the final position estimation is obtained filtering the signal obtained in equation (18) using a low pass filter of second order with $f_{c}=100 \mathrm{~Hz}$.

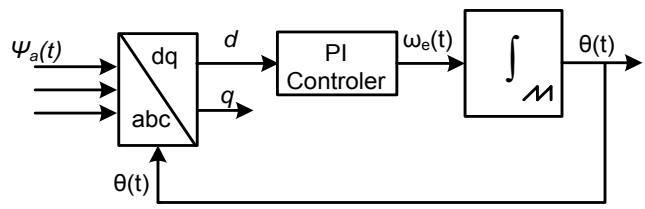

Fig. 5 Synchronous reference frame PLL

\section{7.- Results of the Comparative Study}

The system parameters used in the PSIM $^{\mathrm{TM}}$ simulation are described in Table 1. The studied position and speed estimation techniques have similar behaviors. The study has been carried out for the speed range from 150 to 400 RPM. Figures $6 a$ and $6 b$ show the position estimators performance at step changes of the speed reference. The best performance is achieved by the SKO and PLL techniques. When using EKF and SMO the response is faster but noisier (a higher ripple is observed). The SMO technique has errors in the estimated position signal for various speeds due to the used low-pass filter. The robustness of the position and speed estimators was studied by simulation, with identification errors in the resistance and inductance of the machine. Figures $7 a$ and $7 b$ show the sensitivity to a $\pm 25 \%$ tolerance in $\mathrm{R}_{\mathrm{s}}$ and $\mathrm{L}_{\mathrm{s}}$.
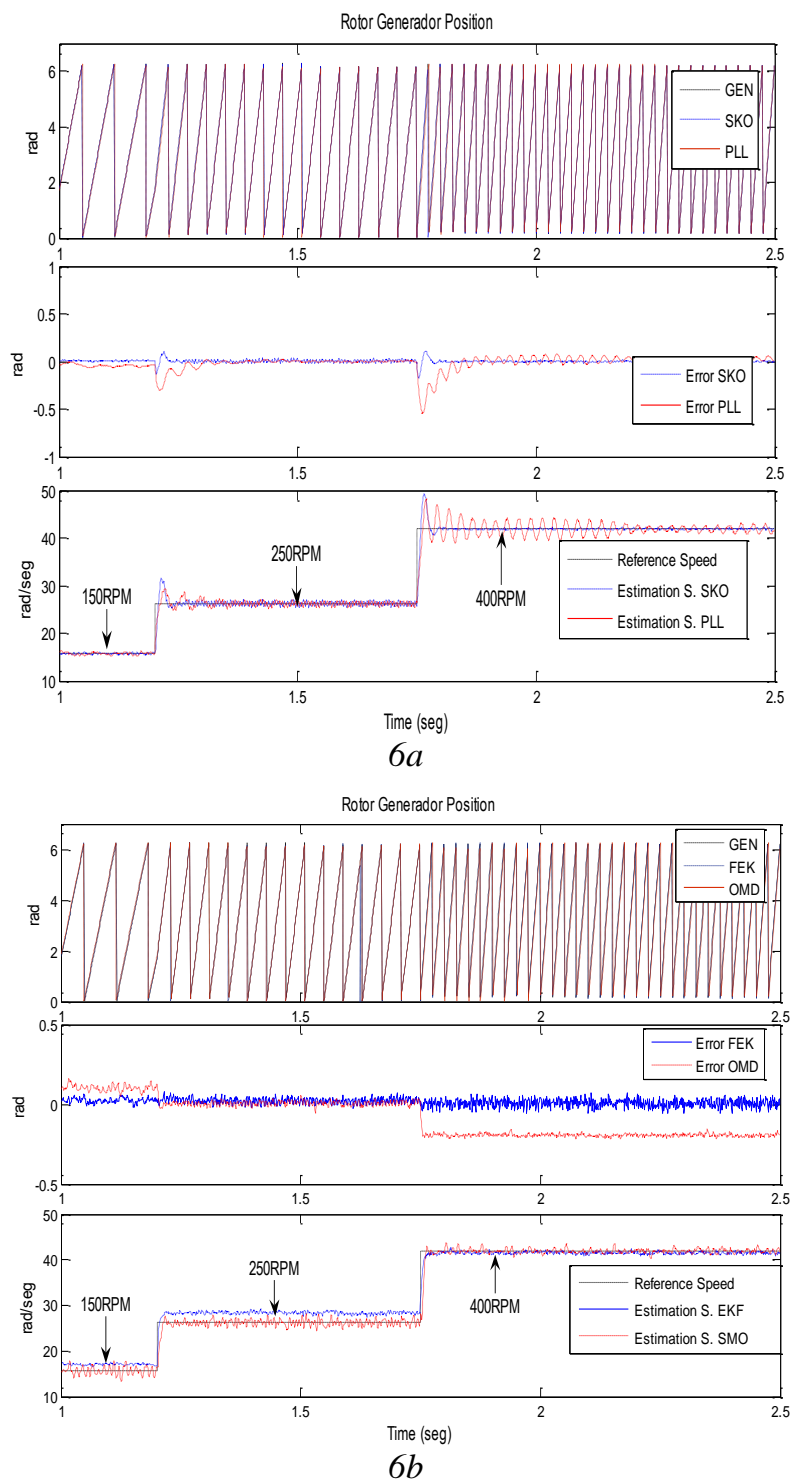

Fig. $6 \mathrm{a}$ and $6 \mathrm{~b}$. Position and speed observer performance at step changes of the speed reference. 


\begin{tabular}{|l|c|}
\hline \multicolumn{2}{|c|}{ Table 1} \\
\hline Number of poles $P$ & 12 \\
\hline Armature resistance and Inductances & $5 \Omega, 25 \mathrm{mh}$ \\
\hline Sampling time $\left(T_{s}\right)$ & $10 \mu \mathrm{seg}$. \\
\hline $\begin{array}{l}\text { Amplitude of the flux linkages } \\
\text { coefficient }\end{array}$ & $\begin{array}{c}0.9022 \\
\text { volt/rad/s }\end{array}$ \\
\hline Speed of the generator maximal & $625 \mathrm{RPM}$ \\
\hline DC link Voltage & $800 \mathrm{volt}$. \\
\hline Inertia coefficient systems & $\begin{array}{c}0.083 \mathrm{~kg}^{*} \mathrm{~m} \\
/ \mathrm{seg}^{2}\end{array}$ \\
\hline
\end{tabular}
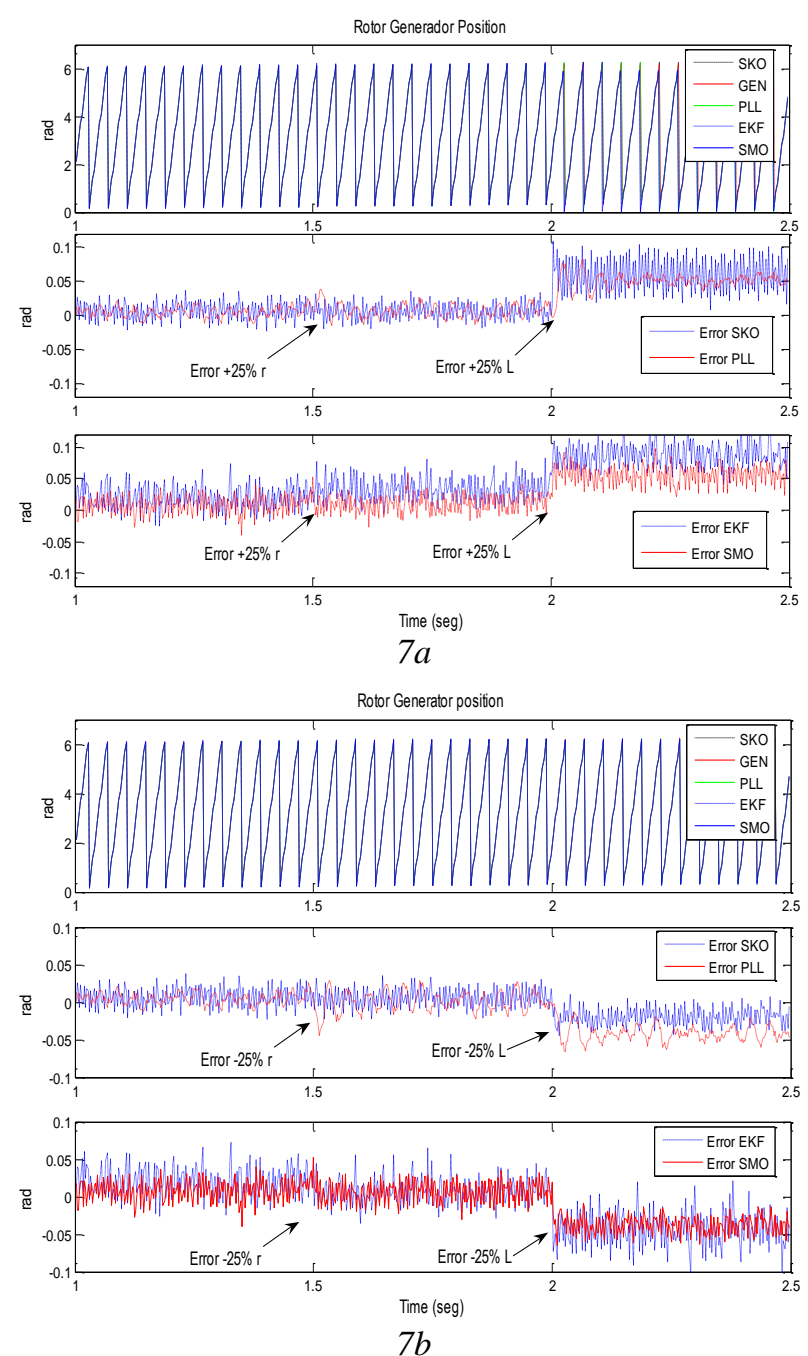

Fig. 7a and 7b, Position observer performance, with errors of $\pm 25 \%$ in $R_{s}$ and $L_{s}$, at $250 R P M$.

\section{8.- Conclusions}

A comparative study of the performance achieved by several estimation techniques for sensorless control of PMSGs has been carried out in this paper. The OSK technique has the best performance among all of the estimation techniques under study, using a simple algorithm which uses a constant gain matrix. The performance of the PLL technique is affected by the tuning parameters, and the SMO technique has an error in the position and speed estimation at different speed operations. Finally the EKF technique has a noisy performance and its implementation is difficult due to mathematical complexities.

\section{References}

[1] Adam M., Xavier R., Frédéric R., "Architecture Complexity and Energy Efficiency of Small Wind Turbines", IEEE Transactions on Industrial Electronics. Vol. 54, Issue 1, Feb. 2007.

[2] Ming Y., Gengyin L., Ming Z., Chengyong Z., "Modeling of the Wind Turbine with a Permanent Magnet Synchronous Generator for Integration", IEEE Power Eng. Society General Meeting, Jun. 2007.

[3] Silverio B., Roberto O., Mauro Z., "Sensorless Full-Digital PMSM Drive With EKF Estimation of Speed and Rotor Position", IEEE Trans. on Ind. Elec., Vol, 46, No.1, Feb. 1999

[4] L. Harnefors,"Speed estimation from noisy resolver signal" in Proc. 6th Int. Conf. Power Electron. And Variable Speed Drives, 1996.

[5] Pedro R., Josep P., Joan B., Ignacio C., Rolando P., Dushan B. "Decoupled Double Synchronous Reference Frame PLL for Power Converters Control", IEEE Trans. on Power Electronics. Vol. 22, Issue 2, March. 2007.

[6] Kittithuch P. Mongkol K.,"Speed Sensorless Control of PMSM Using An Improved Sliding Mode Observer With Sigmoid Function", ECTI Transactions on Electrical ENG., Electronic, and Communications, Vol. 5, No.1, February 2007.

[7] K. Paponpen, M. Konghirun, "An Improved Sliding Mode Observer for Speed Sensorless Vector Control Drive of PMSM" Power electronics and Motion Control Conference, 2006, IPEMC, '06, CES/IEEE, $5^{\text {th }}$ International. 14-15 Aug, 2006.

[8] Changsheng LI., Malik E.," A Robust Sliding Mode Observer for Permanent Magnet Synchronous Motor Drives", Industrial Electronics Society, IEEE 2002 28th Annual Conference. Pub. Vol. 2, Page(s): 1014- 1019.

[9] S. Chi, Z. Zhang, L. Xu," A Novel Sliding Mode Observer with Adaptive Feedback Gain for PMSM Sensorless Vector Control", Power Electronics Specialists Conference, IEEE 2007. 17-21 June 2007, Page(s): 2579-258.

[10] Yong L., Zi Q., David H., "Instantaneous Torque Estimation in Sensorless Direct-Torque-Controlled Brushless DC Motors”, IEEE Transactions on Power Electronics. Applications, Vol. 42, No. 5, Sep. 2006.

[11] Jamal A., Venkata D., Andrew M., "A review of power converter topologies for wind generators" in Elsevier, Science Direct, Renewable Energy 32 (2007) 2369-2385. 